TRANSACTIONS OF THE

AMERICAN MATHEMATICAL SOCIETY

Volume 352, Number 9, Pages 3995-4006

S 0002-9947(00)02478-8

Article electronically published on February 25, 2000

\title{
ANALYTIC TYPES OF PLANE CURVE SINGULARITIES DEFINED BY WEIGHTED HOMOGENEOUS POLYNOMIALS
}

\author{
CHUNGHYUK KANG
}

\begin{abstract}
We classify analytically isolated plane curve singularities defined by weighted homogeneous polynomials $f(y, z)$, which are not topologically equivalent to homogeneous polynomials, in an elementary way. Moreover, in preparation for the proof of the above analytic classification theorem, assuming that $g(y, z)$ either satisfies the same property as the above $f$ does or is homogeneous, then we prove easily that the weights of the above $g$ determine the topological type of $g$ and conversely. So, this gives another easy proof for the topological classification theorem of quasihomogenous singularities in $\mathbb{C}^{2}$, which was already known. Also, as an application, it can be shown that for a given $h$, where $h\left(w_{1}, \ldots, w_{n}\right)$ is a quasihomogeneous holomorphic function with an isolated singularity at the origin or $h\left(w_{1}\right)=w_{1}^{p}$ with a positive integer $p$, analytic types of isolated hypersurface singularities defined by $f+h$ are easily classified where $f$ is defined just as above.
\end{abstract}

\section{INTRODUCTION}

The aim in this paper is to classify analytically isolated plane curve singularities defined by weighted homogeneous polynomials, which are not homogeneous, in an elementary way. It is known by Theorem 2.5 [K1] that some plane curve singularities defined by homogeneous polynomials can be analytically classified in a concrete sense.

Let $f$ and $g$ be holomorphic functions near the origin in $\mathbb{C}^{2}$ with isolated singularity at the origin. For simplicity of notations, if $f$ and $g$ have the same topological type of singularity at the origin, we denote this relation by $f \sim g$. Otherwise, we write $f \not g$. Also, if $f$ and $g$ have the same analytic type of singularity at the origin, then we write $f \approx g$. Otherwise, we write $f \not \approx g$.

Observe that $f=y\left(z+y^{k}\right)$ for any positive integer $k \geq 2$ is weighted homogeneous, which is not homogeneous, but $f \approx z^{2}+y^{2}$. If $f$ is weighted homogeneous, then it can be shown by Lemma 3.4 that $f$ is not homogeneous with $f \not z^{2}+y^{2}$ if and only if $f \not h$ for any homogeneous polynomial $h$. So, if $f \not z^{2}+y^{2}$, by Theorem 3.10 we can classify analytically isolated plane curve singularities defined by weighted homogeneous polynomials $f$, which are not homogeneous, in an easy and concrete way.

Moreover, in preparation for the proof of Theorem 3.10, assuming that either $f$ is weighted homogeneous and nonhomogeneous with $f \nsim z^{2}+y^{2}$, or $f$ is homogeneous,

Received by the editors May 5, 1998.

2000 Mathematics Subject Classification. Primary 32S15, $14 \mathrm{E} 15$.

Supported by MOE, Project No. BSRI-97-1413 and GARC-KOSEF, 1998. Also supported in part by the SNU-97-03-1061. 
then we prove easily by Theorem 3.5 that the weights of $f$ determine the topological type of $f$ and conversely, by the topological classification theorem of plane curve singularites $([\mathrm{L}],[\mathrm{Z2}])$ only. So, this gives another easy proof for the topological classification theorem of quasihomogeneous singularities in $\mathbb{C}^{2}$, which was already proved by $[\mathrm{Yo}-\mathrm{Su}]$.

Also, as an application, it is shown by Corollary 3.13 that for a given $h$, where $h\left(w_{1}, \ldots, w_{m}\right)$ is a quasihomogeneous holomorphic function with an isolated singularity at the origin or $h\left(w_{1}\right)=w_{1}^{p}$ with a positive integer $p$, analytic types of isolated hypersurface singularities defined by $f+h$ are easily classified by analytic types of weighted homogeneous polynomials $f(y, z)$.

\section{KNOWN PRELIMINARIES}

Definition 2.1. Let $V=\left\{z \in \mathbb{C}^{n+1}: f(z)=0\right\}$ and $W=\left\{z \in \mathbb{C}^{n+1}: g(z)=0\right\}$ be germs of complex analytic hypersurfaces with isolated singularity at the origin.

(i) $f$ and $g$ are said to have the same topological type of singularity at the origin if there is a germ at the origin of homeomorphisms $\phi:\left(U_{1}, 0\right) \rightarrow\left(U_{2}, 0\right)$ such that $\phi(V)=W$ and $\phi(0)=0$ where $U_{1}$ and $U_{2}$ are open subsets in $\mathbb{C}^{n+1}$. In this case, denote this relation by $f \sim g$. Otherwise, we write $f \nsim g$.

(ii) $f$ and $g$ are said to have the same analytic type of singularity at the origin if there is a germ at the origin of biholomorphisms $\psi:\left(U_{1}, 0\right) \rightarrow\left(U_{2}, 0\right)$ such that $\psi(V)=W$ and $\psi(0)=0$ where $U_{1}$ and $U_{2}$ are open subsets of $\mathbb{C}^{n+1}$, that is, $f \circ \psi=u g$ where $u$ is a unit in ${ }_{n+1} \mathcal{O}$, the ring of germs of holomorphic functions at the origin in $\mathbb{C}^{n+1}$. Then we write $f \approx g$. If not, we write $f \not z$.

Theorem 2.2 ([B],$\overline{\mathrm{Bu}}],[\mathrm{Z1}])$. Let $f(y, z)$ be irreducible in ${ }_{2} \mathcal{O}$ with an isolated singularity at the origin in $\mathbb{C}^{2}$. Then the curve defined by $f$ at the origin can be described topologically by $y=t^{n}$ and $z=t^{\alpha_{1}}+\cdots+t^{\alpha_{p}}$ where $n<\alpha_{1}<\cdots<\alpha_{p}$ and $n>\left(n, \alpha_{1}\right)>\cdots>\left(n, \alpha_{1}, \ldots, \alpha_{p}\right)=1$. If for a given $f$ there is another homeomorphic parametrization defined by $y=t^{m}$ and $z=t^{\beta_{1}}+\cdots+t^{\beta_{q}}$ where $m<\beta_{1}<\cdots<\beta_{q}$ and $m>\left(m, \beta_{1}\right)>\cdots>\left(m, \beta_{1}, \cdots \beta_{q}\right)=1$, then $n=m, p=q$ and $\alpha_{i}=\beta_{i}$ for $1 \leq i \leq p$. Conversely, the curve defined by the parameter with the same kind of inequality as above must be irreducible at the origin.

Theorem $2.3([\mathrm{~L}], \mathrm{Z2}])$. Let $f(y, z)$ be in ${ }_{2} \mathcal{O}$ with an isolated singularity at the origin in $\mathbb{C}^{2}$. Then the topological type of the plane curve singularity defined by $f$ is determined by the topological type of every irreducible component of $f$ at $O$ and all the pairs of intersection multiplicity of these two components.

Theorem 2.4 ([S]). Quasihomogeneous function with isolated singularity at origin in $\mathbb{C}^{n}$ can be put into weighted homogeneous polynomial by a biholomorphic change of coordinates.

Theorem $2.5([\mathrm{~K} 1])$. Let $f=z^{n}+y^{n}+\sum_{i=1}^{k} a_{i} y^{n-i} z^{i}$ and $g=z^{n}+y^{n}+$ $\sum_{j=1}^{l} b_{j} y^{n-j} z^{j}$ be homogeneous polynomials with isolated singularity at the origin in $\mathbb{C}^{2}$ where $n \geq 2 k+3, n \geq 2 l+3$ and $n \geq 5$. Then $f \approx g$ if and only if there is a complex number $\rho$ with $\rho^{n}=1$ such that $b_{i}=a_{i} \rho^{i}$ for $i=1,2, \ldots, k=l$. Moreover, if $f=z^{4}+a y^{3} z+y^{4}$ and $g=z^{4}+b y^{3} z+y^{4}$ have an isolated singularity at the origin, then $f \approx g$ if and only if $a^{4}=b^{4}$. 


\section{Analytic types of Plane CURVE Singularities DEFined BY WEIGHTED HOMOGENEOUS POLYNOMIALS WHICH ARE NOT HOMOGENEOUS AND ITS APPLICATION}

Let ${ }_{2} \mathrm{O}$ be the ring of convergent power series at the origin in $\mathbb{C}^{2}$.

Definition 3.1. $f \in{ }_{2} \mathcal{O}$ is called a weighted homogeneous polynomial with weights $\left(w_{1}, w_{2}\right)$, where $\left(w_{1}, w_{2}\right)$ are fixed positive rational numbers, if it can be expressed as a linear combination of monomials $z^{\alpha} y^{\beta}$ for which $\frac{\alpha}{w_{1}}+\frac{\beta}{w_{2}}=1$.

Definition 3.2. Let $f \in{ }_{2} \mathcal{O}$ and $g \in{ }_{2} \mathcal{O}$. If $f$ and $g$ have no common component at the origin, the intersection number of $f$ and $g$, denoted by $I(f, g)$, is said to be the dimension of the $\mathbb{C}$-algebra ${ }_{2} \mathcal{O} /(f, g)$ as a complex vector space, where $(f, g)$ is the ideal generated by $f$ and $g$ in ${ }_{2} \mathcal{O}$. Note that $(f, g)=(f, g+h f)$ for any $h \in{ }_{2} \mathcal{O}$.

Throughout this section, we assume that $f$ is a weighted homogeneous polynomial with an isolated singularity at the origin in $\mathbb{C}^{2}$ which are not homogeneous. Then to classify such singularities topologically and analytically in an elementary way, we need the following lemmas.

Lemma 3.3. Let $f$ be a weighted homogeneous polynomial with an isolated singularity at the origin in $\mathbb{C}^{2}$ where $f$ is not a homogeneous polynomial. If $f \neq z^{2}+y^{2}$, then $f$ can be written analytically without loss of generality as follows:

$$
\begin{aligned}
& f(y, z)=y^{\varepsilon_{1}} z^{\varepsilon_{2}} f_{1}(y, z) \text { with } \\
& f_{1}(y, z)=z^{n}+y^{k}+\sum_{i=1}^{d-1} A_{i} y^{i k_{1}} z^{(d-i) n_{1}}
\end{aligned}
$$

where $2 \leq n<k$ and $d=\operatorname{gcd}(n, k)$ with $n=n_{1} d$ and $k=k_{1} d$, each $\varepsilon_{i}$ is either 1 or 0 for $i=1,2$, and all $A_{i}$ are complex numbers for $1 \leq i \leq d-1$, satisfying the property $(*)$.

$$
\text { if } \operatorname{gcd}(n, k)=n \text {, i.e., } n_{1}=1 \text {, then } A_{1} \text { is zero. }
$$

Proof. By assumption, we may put $f$ as follows:

$$
\begin{aligned}
& f(y, z)=y^{\varepsilon_{1}} z^{\varepsilon_{2}} \ell(y, z) \quad \text { with } \\
& \ell(y, z)=B_{0} z^{n}+B_{n} y^{k}+\sum_{i=1}^{n-1} B_{i} y^{\beta_{i}} z^{n-i}
\end{aligned}
$$

where $1 \leq n<k$, each $\varepsilon_{i}$ is either 1 or 0 for $i=1,2$, and all $B_{i}$ are complex numbers for $0 \leq i \leq n$ with $B_{0} B_{n} \neq 0$ and $1<\beta_{1}<\cdots<\beta_{n-1}<k$. Observe that $f(y, z)$ is weighted homogeneous if and only if $\ell(y, z)$ is weighted homogeneous. Let $d=\operatorname{gcd}(n, k)$ with $n=n_{1} d$ and $k=k_{1} d$. Then $\ell(y, z)$ can be rewritten in the form

$$
\ell(y, z)=B_{0} z^{n}+B_{n} y^{k}+\sum_{i=1}^{d-1} B_{i} y^{i k_{1}} z^{(d-i) n_{1}}
$$

Considering $f(a y, b z)$ for some nonzero numbers $a, b$, then it is clear that

$$
\begin{aligned}
& f(y, z) \approx y^{\varepsilon_{1}} z^{\varepsilon_{2}} f_{1}(y, z) \text { with } \\
& f_{1}(y, z)=z^{n}+y^{k}+\sum_{i=1}^{d-1} C_{i} y^{i k_{1}} z^{(d-i) n_{1}}
\end{aligned}
$$


where all $C_{i}$ are complex numbers for $1 \leq i \leq d-1$.

So it is enough to prove analytically by a nonsingular change of coordinates, if necessary, that (i) $n \geq 2$ and (ii) whenever $\operatorname{gcd}(n, k)=n$, the coefficient $C_{1}$ may be eliminated as follows:

(i) To prove that $n \geq 2$, it suffices to consider four subcases below.

(ia) If $n=0$, then it would be impossible.

(ib) If $n=1, \varepsilon_{1}=1$ and $\varepsilon_{2}=0$, then $f \approx y\left(z+y^{k}\right) \approx z^{2}+y^{2}$.

(ic) If $n=1, \varepsilon_{1}=0$ and $\varepsilon_{2}=1$, then $f \approx z\left(z+y^{k}\right) \approx z^{2}+y^{2 k}$, which satisfies the desired property.

(id) If $n=1, \varepsilon_{1}=1$ and $\varepsilon_{2}=1$, then $f \approx y z\left(z+y^{k}\right) \approx y\left(z^{2}+y^{2 k}\right)$, which satisfies the desired property.

(ii) To prove that the coefficient $C_{1}$ may be assumed to be zero after a nonsingular change of coordinates if necessary, suppose that $\operatorname{gcd}(n, k)=n$ with $2 \leq n<k$ and $C_{1} \neq 0$. Otherwise, either $\operatorname{gcd}(n, k)<n$ or $C_{1}=0$ implies that there is nothing to prove. Then it suffices to consider two subcases such as (iia) $\varepsilon_{2}=0$ and (iib) $\varepsilon_{2}=1$ in $f=y^{\varepsilon_{1}} z^{\varepsilon_{2}} f_{1}$ as follows: Note that $n=d$ with $n_{1}=1$.

(iia) Let $\varepsilon_{2}=0$. Recall that $f \approx y^{\varepsilon_{1}} f_{1}(y, z)=y^{\varepsilon_{1}}\left(z^{n}+y^{k}+\sum_{i=1}^{n-1} C_{i} y^{i k_{1}} z^{n-i}\right)$ since $\operatorname{gcd}(n, k)=n$ and $k=n k_{1}$. If $n=2$, then it is clear that $f \approx y^{\varepsilon_{1}}\left(z^{2}+y^{k}\right)$, which satisfies the desired property. So, we may assume that $n \geq 3$. If $n \geq 3$, then by a nonsingular change of coordinates $\phi$ with $\phi(y, z)=\left(y, z-\frac{1}{n} C_{1} y^{k_{1}}\right)$, we have

$$
f \approx y^{\varepsilon_{1}}\left(z^{n}+D_{n} y^{k}+\sum_{i=2}^{n-1} D_{i} y^{i k_{1}} z^{n-i}\right)
$$

where each $D_{i}$ are complex numbers for $2 \leq i \leq n$ and either $D_{n-1}$ or $D_{n}$ is nonzero since $f$ has an isolated singular point. Note that $f \circ \phi$ has the same weights as $f$ does. If $D_{n} \neq 0$, it is clear that $f \approx y^{\varepsilon_{1}}\left(z^{n}+y^{k}+\sum_{i=2}^{n-1} A_{i} y^{i k_{1}} z^{n-i}\right)$ for some complex numbers $A_{i}, 2 \leq i \leq n-1$. If $D_{n}=0$ and $D_{n-1} \neq 0$, then note that $n \geq 3$ and $f \approx y^{\varepsilon_{1}} z\left(z^{n-1}+D_{n-1} y^{(n-1) k_{1}}+\sum_{i=2}^{n-2} D_{i} y^{i k_{1}} z^{n-i-1}\right)$. In this case, it is clear.

(iib) Let $\varepsilon_{2}=1$. Then consider $f \approx y^{\varepsilon_{1}}\left(z f_{1}\right)$ again with

$$
z f_{1}=z^{n+1}+y^{n k_{1}} z+\sum_{i=1}^{n-1} C_{i} y^{i k_{1}} z^{n+1-i} .
$$

Since $n+1 \geq 3$, use the similar method just as we have seen in the subcase (iia), and then the proof is done.

Lemma 3.4. Let $f \in{ }_{2} \mathcal{O}$ and $f$ be weight homogeneous with an isolated singular point at the origin. Then $f$ is not homogeneous with $f \nsim z^{2}+y^{2}$ if and only if $f \not h$ for any homogeneous polynomial $h \in{ }_{2} \mathcal{O}$.

Proof. First, we assume that $f$ is not homogeneous with $f \not z^{2}+y^{2}$. By Lemma $3.3, f$ can be written analytically in the form

$$
\begin{aligned}
f & =y^{\varepsilon_{1}} z^{\varepsilon_{2}} f_{1} \quad \text { with } \\
f_{1} & =z^{n}+y^{k}+\sum_{i=1}^{d-1} A_{i} y^{i k_{1}} z^{(d-i) n_{1}} \\
& =\Pi_{i=1}^{d}\left(z^{n_{1}}+c_{i} y^{k_{1}}\right)
\end{aligned}
$$

where the $c_{i}$ are nonzero distinct complex numbers, satisfying the same properties and notations as in Lemma 3.3. 
Observe that the intersection number of any two distinct irreducible components of a homogeneous polynomial with degree $\geq 2$ is equal to one at the origin in $\mathbb{C}^{2}$. Then, by Theorem 2.3, it is enough to show that either there are at least two irreducible components of $f$, called $\alpha$ and $\beta$, such that $I(\alpha, \beta)>1$, using the notation as in Definition 3.2, or $f$ itself is an irreducible component of $f$ with multiplicity $\geq 2$.

By Theorem 2.3 and the above observation, it is enough to consider the following four cases:

(1) Let $\varepsilon_{1}=\varepsilon_{2}=1$. Then $d \geq 1$ because $f \not z^{2}+y^{2}$, and so $I\left(z, z^{n_{1}}+y^{k_{1}}\right)=$ $k_{1}>n_{1} \geq 1$.

(2) Let $\varepsilon_{1}=1$ and $\varepsilon_{2}=0$. Then there are two subcases: (2a) $d=1$ and (2b) $d \geq 2$.

(2a) If $d=1$, then $n \geq 2$ with $\operatorname{gcd}(n, k)=1$, because $n=1$ implies that $f=y\left(z+y^{k}\right) \sim z^{2}+y^{2}$. Note that $z^{n}+y^{k}$ is an irreducible component of $f$. Then, $I\left(y, z^{n}+y^{k}\right)=n>1$.

(2b) If $d \geq 2$, then $I\left(z^{n_{1}}+c_{1} y^{k_{1}}, z^{n_{1}}+c_{2} y^{k_{1}}\right)=n_{1} k_{1}>1$, since $k_{1}>n_{1} \geq 1$.

(3) Let $\varepsilon_{1}=0$ and $\varepsilon_{2}=1$. Then $d \geq 1$, and so $I\left(z, z^{n_{1}}+y^{k_{1}}\right)=k_{1}>n_{1} \geq 1$.

(4) Let $\varepsilon_{1}=\varepsilon_{2}=0$. Then there are two subcases: (4a) $d=1$ and (4b) $d \geq 2$.

(4a) If $d=1$, then $n \geq 2$ with $\operatorname{gcd}(n, k)=1$. Note that $z^{n}+y^{k}$ is the only one irreducible component of $f$. There is nothing to prove.

(4b) If $d \geq 2$, then $I\left(z^{n_{1}}+c_{1} y^{k_{1}}, z^{n_{1}}+c_{2} y^{k_{2}}\right)=n_{1} k_{1}>1$, since $k_{1}>n_{1} \geq 1$.

By (1), (2), (3) and (4), we showed that $f \nsim h$ for any homogeneous polynomial $h \in{ }_{2} \mathcal{O}$. Next, the converse is trivial. Thus, the proof is done.

Now suppose that $f$ satisfies the property as in Lemma 3.4. Then, depending on the fact that weights $\left(w_{1}, w_{2}\right)$ of $f$ are positive integers or not, we can classify topologically the above singularities by the following.

Theorem 3.5. Let $2 \leq n<k$. Assume that $f(y, z)$ is a weighted homogeneous polynomial with an isolated singular point at the origin in $\mathbb{C}^{2}$ which is not homogeneous. If $f \neq z^{2}+y^{2}$, then $f$ is topologically equivalent to one and only one of the following: Let $d=\operatorname{gcd}(n, k)$.

(I) $f \sim z^{n}+y^{k}$ with weights $(n, k)$.

(i) $d<n$,

(ii) $d=n$.

(II) $f \sim z\left(z^{n}+y^{k}\right)$ with weights $\left(n+1, k+\frac{k}{n}\right)$.

(i) $d<n$.

(III) $f \sim y\left(z^{n}+y^{k}\right)$ with weights $\left(n+\frac{n}{k}, k+1\right)$.

(i) $d<n$,

(ii) $d=n$.

(IV) $f \sim y z\left(z^{n}+y^{k}\right)$ with weights $\left(n+1+\frac{n}{k}, k+1+\frac{k}{n}\right)$.

(i) $d<n$.

In general, suppose that either $f$ satisfies the above assumption with $f \not z^{2}+y^{2}$, or $f$ is homogeneous. Then the weights of $f$ determine the topological type of $f$ and conversely.

Proof. By Lemma 3.3, we may assume analytically that 


$$
\begin{aligned}
f(y, z) & =y^{\varepsilon_{1}} z^{\varepsilon_{2}} f_{1}(y, z) \text { with } \\
f_{1}(y, z) & =z^{n}+y^{k}+\sum_{i=1}^{d-1} A_{i} y^{i k_{1}} z^{(d-i) n_{1}} \\
& =\prod_{i=1}^{d}\left(z^{n_{1}}+c_{i} y^{k_{1}}\right)
\end{aligned}
$$

where $d=\operatorname{gcd}(n, k)$ with $n=n_{1} d$ and $k=k_{1} d$, and $\varepsilon_{1}, \varepsilon_{2}$ are either 1 or 0 , respectively and the $c_{i}$ are distinct nonzero numbers, and if $\operatorname{gcd}(n, k)=n$, then $A_{1}$ is zero. For the proof, let $g(y, z)$ be a weighted homogeneous polynomial with an isolated singular point at the origin in $\mathbb{C}^{2}$, which is not homogeneous. If $g \nsim z^{2}+y^{2}$, then by Lemma 3.3, $g$ can be written analytically in the form

$$
\begin{aligned}
g(y, z) & =y^{\delta_{1}} z^{\delta_{2}} g_{1}(y, z) \quad \text { with } \\
g_{1}(y, z) & =z^{m}+y^{l}+\sum_{j=1}^{e-1} B_{j} y^{j l_{1}} z^{(e-j) m_{1}} \\
& =\Pi_{j=1}^{e}\left(z^{m_{1}}+r_{j} y^{l_{1}}\right)
\end{aligned}
$$

where $2 \leq m<l, e=\operatorname{gcd}(m, l)$ with $m=m_{1} e$ and $l=l_{1} e$, and $\delta_{1}, \delta_{2}$ are either 1 or 0 , respectively, and all $B_{j}$ are complex numbers for $1 \leq j \leq e-1$, and all $r_{j}$ are distint nonzero complex numbers for $1 \leq j \leq e$.

For brevity of notation, if $f \in{ }_{2} \mathcal{O}$ has an isolated singularity at the origin in $\mathbb{C}^{2}$, define $S(f)$ by the set of intersection numbers of any two irreducible components of $f$.

To prove the theorem, it is enough to consider the following three cases: (1) $d=1,(2) 2 \leq d<n$, and (3) $d=n$.

(1) Let $d=1$. Then $\operatorname{gcd}(n, k)=1$, and so $f=y^{\varepsilon_{1}} z^{\varepsilon_{2}}\left(z^{n}+y^{k}\right)$. Assume that $f \sim g$. By Theorem 2.3, $z^{n}+y^{k}$ is topologically equivalent to only one irreducible component of $g$ because $n \geq 2$ and $\operatorname{gcd}(n, k)=1$. Then, $m_{1} \geq 2$ and $1=e=\operatorname{gcd}(m, l)$ by Theorem 2.3. So, $z^{n}+y^{k} \sim z^{m}+y^{l}$. By Theorem 2.3, $n=m, k=l$ and also $g=y^{\delta_{1}} z^{\delta_{2}}\left(z^{n}+y^{k}\right)$ because $n<k$ and $m<l$. Note that $S(f)=\left\{\varepsilon_{1} \varepsilon_{2}, \varepsilon_{1} n, \varepsilon_{2} k\right\}-\{0\}$ and $S(g)=\left\{\delta_{1} \delta_{2}, \delta_{1} n, \delta_{2} k\right\}-\{0\}$. Since $1<n<k$ and $S(f)=S(g)$ by Theorem 2.3, then it is trivial to prove that $\delta_{1}=\varepsilon_{1}$ and $\delta_{2}=\varepsilon_{2}$. Thus, we proved that $\varepsilon_{1}, \varepsilon_{2}, n, k$ are topologically invariant.

(2) Let $2 \leq d<n$. Note that $n=n_{1} d$ and $k=k_{1} d$ with $\operatorname{gcd}(n, k)=d$. Then $k_{1}>n_{1} \geq 2$, because $n_{1}=1$ implies that $n=n_{1} d=d$ and so it is a contradiction. So, $f=y^{\varepsilon_{1}} z^{\varepsilon_{2}} \prod_{i=1}^{d}\left(z^{n_{1}}+c_{i} y^{k_{1}}\right)$ with $n_{1} \geq 2$ and $d \geq 2$. Assume that $f \sim g$. By Theorem 2.3, $z^{n_{1}}+y^{k_{1}}$ is topologically equivalent to an irreducible component of $g$ because $\operatorname{gcd}\left(n_{1}, k_{1}\right)=1$. Since $n_{1} \geq 2$, then $m_{1} \geq 2$. For example, since $z^{n_{1}}+c_{1} y^{k_{1}} \sim z^{n_{1}}+c_{2} y^{k_{1}}$ and $z^{m_{1}}+r_{1} y^{l_{1}} \sim z^{m_{1}}+r_{2} y^{l_{1}}$, then $n_{1}=m_{1}$ and $k_{1}=l_{1}$, and so $d=e$ by Theorem 2.3, counting the number of irreducible components with multiplicity $\geq 2$. That is, $n=m, k=l$ and then $g=y^{\delta_{1}} z^{\delta_{2}} \prod_{i=1}^{d}\left(z^{n_{1}}+r_{i} y^{k_{1}}\right)$. Note that $S(f)=\left\{\varepsilon_{1} \varepsilon_{2}, \varepsilon_{1} n_{1}, \varepsilon_{2} k_{1}, n_{1} k_{1}\right\}-\{0\}$ and $S(g)=\left\{\delta_{1} \delta_{2}, \delta_{1} n_{1}, \delta_{2} k_{1}, n_{1} k_{1}\right\}-\{0\}$. Since $S(f)=S(g)$ and $1<n_{1}<k_{1}<n_{1} k_{1}$, then $\varepsilon_{1}=\delta_{1}$ and $\varepsilon_{2}=\delta_{2}$. Thus, we proved that $\varepsilon_{1}, \varepsilon_{2}, n$ and $k$ are topologically invariant.

(3) Let $d=n$. Then $k_{1}>n_{1}=1$ because $n=n_{1} d=n_{1} n$ and $k=k_{1} d=k_{1} n>$ $n$. Then $f=y^{\varepsilon_{1}} z^{\varepsilon_{2}} \prod_{i=1}^{n}\left(z+c_{i} y^{k_{1}}\right)$. If $\varepsilon_{2}=0$, then $n \geq 2$ because $f \not z^{2}+y^{2}$. If $\varepsilon_{2}=1$, then $f=y^{\varepsilon_{1}} z \prod_{i=1}^{n}\left(z+c_{i} y^{k_{1}}\right)$ with $n \geq 1$ is topologically equivalent to $y^{\varepsilon_{1}} \Pi_{i=1}^{n+1}\left(z+c_{i} y^{k_{1}}\right)$ where the $c_{n+1}$ is a nonzero number by Theorem 2.3. So, we may assume without loss of generality that $f=y^{\varepsilon_{1}} \Pi_{i=1}^{n}\left(z+c_{i} y^{k_{1}}\right)$ with $n \geq 2$. If $f \sim g$, 
then by Theorem 2.3, $m_{1}=1$ and $e=m$ because any irreducible component of $f$ has no singular point at the origin. So, we may assume that $g=y^{\delta_{1}} \Pi_{j=1}^{m}\left(z+r_{j} y^{l_{1}}\right)$ with $m \geq 2$ because $g \not z^{2}+y^{2}$, by a similar argument as in $f$. Note that $S(f)=\left\{\varepsilon_{1}, k_{1}\right\}-\{0\}$ and $S(g)=\left\{\delta_{1}, l_{1}\right\}-\{0\}$. By Theorem 2.3, $S(f)=S(g)$, and so $k_{1}=l_{1}$ and $\varepsilon_{1}=\delta_{1}$ because $k_{1}>1$ and $l_{1}>1$. Considering the number of irreducible components of $f$ and $g$ respectively, then $\varepsilon_{1}=\delta_{1}$ implies that $n=m$ by Theorem 2.3. So, $k=l$ because $k=k_{1} d=k_{1} n$ and $l=l_{1} e=l_{1} m=k_{1} n$. Thus, we proved that $\varepsilon_{1}, n$ and $k$ are topologically invariant, assuming that $\varepsilon_{2}=\delta_{2}=0$.

Note that we do not assume any restriction on the integer $\operatorname{gcd}(m, l)=e$ for the proof of (1), (2) and (3), respectively. So, if $\operatorname{gcd}(n, k)<n$, then (1) and (2) have the different topological types by Theorem 2.3, counting the number of irreducible components, each of which has a multiplicity $p$ with $p \geq 2$. Also, by Theorem 2.3, (3) is topologically different from both (1) and (2) because there is no irreducible component with multiplicity $\geq 2$ in (3). Therefore, by (1), (2) and (3), the topological classification of singularities defined by the above weighted homogeneous polynomials is done.

Also, by using the numbers $\frac{k}{n} \geq 1, \frac{n}{k} \leq 1$ and $\operatorname{gcd}(n, k)$, we showed that the weights of $f$ and $g$ are equal if and only if $f \sim g$, together with the weights of homogeneous polynomials. Thus, this completes the proof.

Considering weights of a homogeneous polynomial, then by Theorem $2.4[\mathrm{~S}]$ and Theorem 3.5, we prove easily the following:

Theorem 3.6 ([Yo-Su] $)$. The topology of a quasihomogeneous singularity in $\mathbb{C}^{2}$ determines the weights of the polynomial defining the singularity.

Theorem 3.7. Let $2 \leq n<k$ and $d=\operatorname{gcd}(n, k)<n$ with $n=n_{1} d$ and $k=k_{1} d$. Assume that $f \sim g \sim z^{n}+y^{k}$ at the origin where $f$ and $g$ are weighted homogeneous polynomials with isolated singularity at the origin in $\mathbb{C}^{2}$. Then $f$ and $g$ may be written analytically as follows:

$$
\begin{aligned}
& f=z^{n}+y^{k}+\sum_{i=1}^{d-1} A_{i} y^{i k_{1}} z^{(d-i) n_{1}} \quad \text { and } \\
& g=z^{n}+y^{k}+\sum_{i=1}^{d-1} B_{i} y^{i k_{1}} z^{(d-i) n_{1}}
\end{aligned}
$$

where the $A_{i}$ and $B_{i}$ are complex numbers for $1 \leq i \leq d-1$. As a conclusion, $f \approx g$ if and only if there is a complex number $\omega$ with $\omega^{d}=1$ such that $A_{i} \omega^{i}=B_{i}$ for $i=1, \ldots, d-1$.

Proof. If $f \sim g \sim z^{n}+y^{k}$ with $2 \leq n<k$ and $d<n$, it is clear that $f$ and $g$ have the above representation by Lemma 3.3 and Theorem 3.5. If $d=1$, there is nothing to prove. So we may assume that $1<d<n$. Suppose that $f \approx g$. Then by definition, there is a biholomorphic mapping $\phi:\left(U_{1}, 0\right) \rightarrow\left(U_{2}, 0\right)$ such that $f \circ \phi=u g$ where $U_{1}$ and $U_{2}$ are open subsets in $\mathbb{C}^{2}$ containing the origin and $u$ is a unit in ${ }_{2} \mathcal{O}$. Write $\phi(y, z)=(L, H)$ as follows:

$$
\begin{aligned}
& H=H(y, z)=a_{10} z+a_{01} y+H_{2}+H_{3}+\cdots \quad \text { and } \\
& L=L(y, z)=b_{10} z+b_{01} y+L_{2}+L_{3}+\cdots
\end{aligned}
$$


where $H_{r}=H_{r}(y, z)$ and $L_{r}=L_{r}(y, z)$ are homogeneous polynomials of degree $r$ with $H_{r}=\sum_{q=0}^{p+q=r} a_{p q} z^{p} y^{q}$ and $L_{r}=\sum_{q=0}^{p+q=r} b_{p q} z^{p} y^{q}$. Then

$$
\begin{aligned}
f \circ \phi(y, z) & =H^{n}+L^{k}+\sum_{i=1}^{d-1} A_{i} L^{i k_{1}} H^{(d-i) n_{1}} \\
& =u\left(z^{n}+y^{k}+\sum_{i=1}^{d-1} B_{i} y^{i k_{1}} z^{(d-i) n_{1}}\right)
\end{aligned}
$$

for some unit $u$ in ${ }_{2} \mathcal{O}$.

Note that $n<k_{1}+(d-1) n_{1}<2 k_{1}+(d-2) n_{1}<\cdots<k, a_{10} b_{01}-a_{01} b_{10} \neq 0$ and then $a_{01}=0$, considering the coefficient of a monomial $y^{n}$ in $f \circ \phi=u g$.

For notation, if the coefficient of monomial $y^{\alpha} z^{\beta}$ in the convergent power series expansion of $h(y, z)$ at the origin is zero where $h=h(y, z)$ is in ${ }_{2} \mathcal{O}$, then we write $y^{\alpha} z^{\beta} \notin h$. Otherwise, we write $y^{\alpha} z^{\beta} \in h$. Let $t$ be the smallest positive integer such that $H_{t}$ cannot be divisible analytically by $z$ if it exists. Otherwise, define $t$ by $+\infty$. Then $t \geq k_{1}+(d-1) n_{1}-n+1=k_{1}-n_{1}+1>\frac{k_{1}}{n_{1}}=\frac{k}{n}$ because $\left(k_{1}-n_{1}+1\right) n_{1}-k_{1}=\left(k_{1}-n_{1}\right)\left(n_{1}-1\right)>0$.

Now, we are going to prove the following cases, respectively.

(I)

(i) To show that $y^{i k_{1}} z^{(d-i) n_{1}} \notin H^{n}$ for each $i=1, \ldots, d$, it is enough to prove that $(d-i) n_{1}+\left[n-(d-i) n_{1}\right] t>i k_{1}+(d-i) n_{1}$, that is, $i t n_{1}>i k_{1}$. It is trivial.

(ii) To show that $y^{i k_{1}} z^{(d-i) n_{1}} \notin L^{k}$ for each $i=1, \ldots, d-1$, it is enough to prove that $i k_{1}+(d-i) n_{1}<k$. It is clear.

(iii) To show that $y^{i k_{1}} z^{(d-i) n_{1}} \notin L^{j k_{1}} H^{(d-j) n_{1}}$ for each $i \neq j$, then we need to consider two cases, i.e., $j>i$ and $j<i$. If $j>i$, then it is clear because $j k_{1}+(d-j) n_{1}-\left[i k_{1}+(d-i) n_{1}\right]=(j-i)\left(k_{1}-n_{1}\right)>0$. If $j<i$, then it is enough to prove that $(d-i) n_{1}+\left[(d-j) n_{1}-(d-i) n_{1}\right] t+j k_{1}>i k_{1}+(d-i) n_{1}$, that is, $(i-j)\left(n_{1} t-k_{1}\right)>0$, which is clear.

(II)

(i) To show that $y^{k} \notin H^{n}$, it is enough to prove that $t n>k$, which is trivial.

(ii) To show that $y^{k} \notin L^{i k_{1}} H^{(d-i) n_{1}}$ for each $i=1, \ldots, d-1$, then, it is enough to prove that $i k_{1}+(d-i) n_{1} t>k$, that is, $(d-i)\left(n_{1} t-k_{1}\right)>0$. It is trivial.

Also, it is clear that $z^{n} \in H^{n}, y^{i k_{1}} z^{(d-i) n_{1}} \in L^{i k_{1}} H^{(d-i) n_{1}}$ and $y^{k} \in L^{k}$. Therefore, if we write $u_{0}=u(0,0) \neq 0$ from $f \circ \phi=u g$, then we get the equations as follows: Note that $\left\{i: A_{i}\right.$ is nonzero $\}=\left\{j: B_{j}\right.$ is nonzero $\}$ from $u^{-1} f=g \circ \phi^{-1}$.

$$
a_{10}^{n}=u_{0}, b_{01}^{k}=u_{0} \text { and } A_{i} b_{01}^{i k_{1}} a_{10}^{(d-i) n_{1}}=u_{0} B_{i} \text { for } i=1, \ldots, d-1 .
$$

So, we get that $A_{i} \omega^{i}=B_{i}$ where $\omega=\frac{b_{01}^{k_{1}}}{a_{10}^{n_{1}}}$ with $\omega^{d}=1$.

Conversely, suppose that there is a complex number $\omega$ with $\omega^{d}=1$ such that $A_{i} \omega^{i}=B_{i}$ for $i=1, \ldots, d-1$. Then use the mapping $\phi$ defined by $\phi(y, z)=\left(\omega_{0} y, z\right)$ for some number $\omega_{0}$ such that $\omega_{0}^{k_{1}}=\omega$, and so it is done.

Corollary 3.8. Let $f$ and $g$ be weighted homogeneous with isolated singularity at the origin in $\mathbb{C}^{2}$. Assume that $f \sim y^{\varepsilon_{1}} z^{\varepsilon_{2}}\left(z^{n}+y^{k}\right)$ and $g \sim y^{\delta_{1}} z^{\delta_{2}}\left(z^{m}+y^{l}\right)$ where 
$\varepsilon_{1}, \varepsilon_{2}, \delta_{1}$ and $\delta_{2}$ are either 1 or 0 , respectively, and $2 \leq n<k, d=\operatorname{gcd}(n, k)<n$, and $2 \leq m<l, e=\operatorname{gcd}(m, l)<m$. Then $f$ and $g$ can be analytically written as follows:

$$
\begin{aligned}
f & =y^{\varepsilon_{1}} z^{\varepsilon_{2}} f_{1} \text { with } \\
f_{1} & =z^{n}+y^{k}+\sum_{i=1}^{d-1} A_{i} y^{i k_{1}} z^{(d-i) n_{1}} \text { and } \\
g & =y^{\delta_{1}} z^{\delta_{2}} g_{1} \text { with } \\
g_{1} & =z^{m}+y^{l}+\sum_{j=1}^{e-1} B_{j} y^{j l_{1}} z^{(e-j) m_{1}}
\end{aligned}
$$

where $n=n_{1} d, k=k_{1} d$ and $m=m_{1} e, l=l_{1} e$ and all $A_{i}$, all $B_{j}$ are complex numbers for $1 \leq i \leq d-1,1 \leq j \leq e-1$.

As a conclusion, we get the following:

$f \approx g$ if and only if $\varepsilon_{i}=\delta_{i}$ for $i=1,2$ and $f_{1} \approx g_{1}$ if and only if $\varepsilon_{i}=\delta_{i}$ for $i=1,2$ and $n=m, k=l$ and there is a complex number $\omega$ with $\omega^{d}=1$ such that $A_{i} \omega^{i}=B_{i}$ for $i=1, \ldots d-1$.

Proof. Suppose that $\varepsilon_{i}=\delta_{i}$ for $i=1,2$ and $f_{1} \approx g_{1}$. Then, by Theorem 3.7 it is clear. To prove the converse, assume that $f \approx g$. Then it is enough to consider the following cases: (i) $\varepsilon_{1}=\varepsilon_{2}=1$, (ii) $\varepsilon_{1}=1$ and $\varepsilon_{2}=0$, (iii) $\varepsilon_{1}=0$ and $\varepsilon_{2}=1$.

Case (i). Let $\varepsilon_{1}=\varepsilon_{2}=1$. Since $\operatorname{gcd}(n, k)<n$, then by Theorem $3.5 \varepsilon_{i}=\delta_{i}$ for $i=1,2$, and also $n=m, k=l$. To prove it, using the same notation for $f \circ \phi=u g$ as we have seen in the proof of Theorem 3.7, then

$$
\begin{aligned}
f \circ \phi & =L H\left(f_{1} \circ \phi\right)=L H\left(H^{n}+L^{k}+\sum_{i=1}^{d-1} A_{i} L^{i k_{1}} H^{(d-i) n_{1}}\right) \\
& =u y z g_{1}=u y z\left(z^{n}+y^{k}+\sum_{i=1}^{d-1} B_{i} y^{i k_{1}} z^{(d-i) n_{1}}\right)
\end{aligned}
$$

where $H=a_{10} z+a_{01} y+H_{2}+H_{3}+\cdots$ and $L=b_{10} z+b_{01} y+L_{2}+L_{3}+\cdots$, and $u$ is a unit in ${ }_{2} \mathcal{O}$. Now, we claim that $a_{01}=b_{10}=0$.

Considering the coefficients of monomials $y^{n+2}, z^{n+2}$ and $y z^{n+1}$, respectively, it follows from the above equation that

$$
a_{01}^{n+1} b_{01}=0, a_{10}^{n+1} b_{10}=0,\left(a_{10} b_{01}+(n+1) a_{01} b_{10}\right) a_{10}^{n} \neq 0 .
$$

Since $a_{10} \neq 0$, then $b_{10}=0$ and so $a_{10} b_{01}+(n+1) a_{01} b_{10}=a_{10} b_{01} \neq 0$. Thus, we get that $a_{01}=0$. Note that $a_{10} b_{01}-a_{01} b_{10}=a_{10} b_{01} \neq 0$. Therefore, $L H$ can be rewritten in the form

$$
L H=a_{10} b_{01} y z+\text { monomials of multiplicity } \geq 3 \text { in }{ }_{2} \mathcal{O} .
$$

To prove Case (i), use the same method with the same kind of integer $t$ as we have seen in the proof of Theorem 3.7. Then we can prove that for $0 \leq i \leq d$ each nonzero monomial $y^{i k_{1}+1} z^{(d-i) n_{1}+1}$ belongs exactly to $L^{i k_{1}+1} H^{(d-i) n_{1}+1}$, and so we get the same result as before: Write $u_{0}^{\prime}=\frac{u_{0}}{a_{10} b_{01}}$ where $u_{0}=u(0,0)$. 


$$
a_{10}^{n}=u_{0}^{\prime}, b_{01}^{k}=u_{0}^{\prime}, A_{i} b_{01}^{i k_{1}} a_{10}^{(d-i) n_{1}}=u_{0}^{\prime} B_{i} \text { for } i=1, \ldots, d-1 .
$$

By Theorem 3.7 again, $f_{1} \approx g_{1}$ and then we have proved Case (i).

To prove other cases, use the same technique as in the proof of Case (i) and so it is done.

Theorem 3.9. Let $f$ and $g$ be weighted homogeneous with isolated singularity at the origin in $\mathbb{C}^{2}$. Assume that $f \sim y^{\varepsilon_{1}} z^{\varepsilon_{2}}\left(z^{n}+y^{k}\right)$ and $g \sim y^{\delta_{1}} z^{\delta_{2}}\left(z^{m}+y^{l}\right)$ where $\varepsilon_{1}, \varepsilon_{2}, \delta_{1}$ and $\delta_{2}$ are either 1 or 0 , respectively, and $2 \leq n<k, n=\operatorname{gcd}(n, k)$, and $2 \leq m<l, m=\operatorname{gcd}(m, l)$. Then $f$ and $g$ can be analytically written as follows:

$$
\begin{aligned}
& f=y^{\varepsilon_{1}} z^{\varepsilon_{2}} f_{1} \text { with } \\
& f_{1}=z^{n}+y^{k}+\sum_{i=2}^{n-1} A_{i} y^{i k_{1}} z^{n-i} \text { and } \\
& g=y^{\delta_{1}} z^{\delta_{2}} g_{1} \text { with } \\
& g_{1}=z^{m}+y^{l}+\sum_{j=2}^{m-1} B_{j} y^{j l_{1}} z^{m-j}
\end{aligned}
$$

where $k=k_{1} n, l=l_{1} m$ and all $A_{i}$, all $B_{j}$ are complex numbers for $2 \leq i \leq n-1$, $2 \leq j \leq m-1$. Recall that both $A_{1}$ and $B_{1}$ are zero.

As a conclusion, we get the following: Note that $\operatorname{gcd}(n, k)=n$.

(i) The above $f$ is topologically different from the types which we have seen in Corollary 3.8 .

(ii) $f \approx g$ if and only if $\varepsilon_{i}=\delta_{i}$ for $i=1,2$ and $f_{1} \approx g_{1}$ if and only if $\varepsilon_{i}=\delta_{i}$ for $i=1,2$ and $n=m, k=l$ and there is a complex number $\omega$ with $\omega^{n}=1$ such that $A_{i}=\omega^{i} B_{i}$ for $i=2, \ldots, n-1$.

Proof. (i) It follows from Theorem 2.3, Lemma 3.3 and Theorem 3.5.

(ii) To prove it, suppose that $\varepsilon_{i}=\delta_{i}$ for $i=1,2$ and $f_{1} \approx g_{1}$. Then use the same method and notation for $f \circ \phi=u g$ with the same kind of integer $t$ as we have seen in the proof of Theorem 3.7 and Corollary 3.8. Note that $t \geq 2 k_{1}+n-2-n+1>$ $k_{1}=\frac{k}{n}$ because $A_{1}$ is zero. Then apply the same technique to this case as we have seen in the proof of Corollary 3.8, and so we can get the desired result. To prove the converse, assume that $f \approx g$. Then it is enough to consider the following cases: (a) $\varepsilon_{1}=\varepsilon_{2}=1$, (b) $\varepsilon_{1}=1$ and $\varepsilon_{2}=0$, and (c) $\varepsilon_{1}=0$ and $\varepsilon_{2}=1$.

Case (a). To prove it, use the same method and notation for $f \circ \phi=u g$ as we have seen in the proof of Corollary 3.8. Then it can be shown that $\varepsilon_{i}=\delta_{i}$ for $i=1,2$ and $f_{1} \approx g_{1}$, that is, $\varepsilon_{i}=\delta_{i}$ for $i=1,2$ and there is a complex number $\omega$ with $\omega^{n}=1$ such that $A_{i} \omega^{i}=B_{i}$ for $i=2, \ldots, n-1$.

To prove other cases, use the same technique as in the proof of Case (a) and so it can be done.

Remark 3.9.1. If $2 \leq n<k$ and $\operatorname{gcd}(n, k)=n$, then we have proved by Theorem 3.9 that $z\left(z^{n}+y^{k}\right) \not \approx z^{n+1}+y^{k+\frac{k}{n}}$ and $y z\left(z^{n}+y^{k}\right) \not \approx y\left(z^{n+1}+y^{k+\frac{k}{n}}\right)$. But note that $z\left(z^{n}+y^{k}\right) \sim z^{n+1}+y^{k+\frac{k}{n}}$ and $y z\left(z^{n}+y^{k}\right) \sim y\left(z^{n+1}+y^{k+\frac{k}{n}}\right)$.

Theorem 3.10. Let $f$ and $g$ be weighted homogeneous polynomials, which are not homogeneous, with isolated singularity at the origin in $\mathbb{C}^{2}$ such that $f \neq z^{2}+y^{2}$ 
and $g \nsim z^{2}+y^{2}$. Then we may assume without loss of generality that analytically,

$$
\begin{aligned}
f & =y^{\varepsilon_{1}} z^{\varepsilon_{2}} f_{1} \quad \text { with } \\
f_{1} & =z^{n}+y^{k}+\sum_{i=1}^{d-1} A_{i} y^{i k_{1}} z^{(d-i) n_{1}}, \\
g & =y^{\delta_{1}} z^{\delta_{2}} g_{1} \quad \text { with } \\
g_{1} & =z^{m}+y^{l}+\sum_{j=1}^{e-1} B_{j} y^{j l_{1}} z^{(e-j) m_{1}}
\end{aligned}
$$

where

(a) $2 \leq n<k, d=\operatorname{gcd}(n, k)$ with $n=d n_{1}$ and $k=d k_{1}$,

(b) $2 \leq m<l, e=\operatorname{gcd}(m, l)$ with $m=e m_{1}$ and $l=e l_{1}$,

(c) $\varepsilon_{1}, \varepsilon_{2}, \delta_{1}, \delta_{2}$ are either 1 or 0 , respectively, and

(d) $A_{i}$ and $B_{j}$ are complex numbers for $1 \leq i \leq d-1$ and $1 \leq j \leq e-1$.

Also, we need to assume without loss of generality that

$$
\begin{aligned}
& \text { if } \operatorname{gcd}(n, k)=n, \quad \text { i.e., } \quad n_{1}=1, \text { then } A_{1}=0 \text { and } \\
& \text { if } \operatorname{gcd}(m, l)=m, \quad \text { i.e., } \quad m_{1}=1, \text { then } B_{1}=0 .
\end{aligned}
$$

As a conclusion, we get the following:

$f \approx g$ if and only if $\varepsilon_{i}=\delta_{i}$ for $i=1,2$ and $f_{1} \approx g_{1}$ if and only if $\varepsilon_{i}=\delta_{i}$ for $i=1,2$ and $n=m, k=l$ and there is a complex number $\rho$ with $\rho^{d}=1$ such that $A_{i} \rho^{i}=B_{i}$ for $i=1, \ldots d-1$.

Proof. It follows from Theorem 3.7, Corollary 3.8 and Theorem 3.9.

Definition $3.11(\underline{\underline{S}}])$. Let $f\left(z_{1}, \ldots, z_{n}\right)$ be a holomorphic function with an isolated singular point at the origin in $\mathbb{C}^{n}$. $f$ is called a quasihomogeneous function if $f \approx g$ for some weighted homogeneous polynomial $g$ with an isolated singular point at the origin in $\mathbb{C}^{n}$.

Theorem 3.12 (Ya $)$. Let $f\left(z_{1}, \ldots, z_{n}\right)$ and $g\left(z_{1}, \ldots, z_{n}\right)$ be holomorphic functions with isolated singularity at the origin in $\mathbb{C}^{n}$, and let $h\left(w_{1}, \ldots, w_{m}\right)$ be a quasihomogeneous holomorphic function with an isolated singularity at the origin in $C^{m}$. Then $f \approx g$ at the origin in $\mathbb{C}^{n}$ if and only if $f+h \approx g+h$ at the origin in $\mathbb{C}^{n+m}$.

As an application, by Theorem 3.10 and 3.12 we get

Corollary 3.13. Let $f(y, z)$ and $g(y, z)$ be weighted homogeneous polynomials, satisfying the same assumption and representation as in Theorem 3.10, and let $h$ be defined as in Theorem 3.12 or $h\left(w_{1}\right)=w_{1}^{p}$ for a positive integer $p$. Then $f+h \approx g+h$ if and only if $\varepsilon_{i}=\delta_{i}$ for $i=1,2$ and $n=m, k=l$ and there is a complex number $\rho$ with $\rho^{d}=1$ such that $A_{i} \rho^{i}=B_{i}$ for $i=1, \ldots, d-1$.

\section{REFERENCES}

[A] V.I. Arnold, Normal forms of functions in neighborhoods of degenerate critical points, Russian Math. Surveys, 29, 10-50 (1974). MR 58:24324

[Br] Brauner, K., Zur Geometrie der Funktionen Zweier Komplexen Vesänderlicken, Abh. Math. Sem. Hamburg, 6, 1-54 (1928).

[Bu] Burau, W., Kennzeichung der Schlauchknoten, Abh. Math. Sem. Hamburg, 9, 125-133 (1932). 
[K1] Kang, C., Analytic classification of plane curve singularities defined by some homogeneous polynomials, J. Korean Math. Soc. 30, No.2, 385-397 (1993). MR 94g:14015

[K2] Kang, C., Topological classification of irreducible plane curve singularities in terms of Weierstrass polynomials, Proc. A.M.S. 123, No.5, 1363-1371 (1995). MR 95f:14048

[L] Lejeune, M., Sur l'équivalence des singularité des courbes algébrö̈des planes, Coefficients de Newton, Centre de Math. del 1'Ecole Polytechnique, 1969.

[S] Saito, K., Quasihomogene isolierte Singularitäten von Hyperflächen, Invent. Math. 14, 123142 (1971). MR 45:3767

[Ya] Yau, S. S.-T, A remark on moduli of complex hypersurfaces, Amer. J. Math. 113, 287-292 (1991). MR 92g:32063

[Yo-Su] Yoshinaga, E. and Suzuki, M., Topological types of quasihomogeneous singularities in $\mathbb{C}^{2}$, Topology 18, 113-116 (1979). MR 80k:32017

[Z1] Zariski, O, On the topology of algebroid singularities, Amer. J. Math. 54, 433-465 (1932).

[Z2] Zariski, O, Studies in equisingularity I: Equivalent singularities of plane algebroid curves, Amer. J. Math. 87, 507-536 (1965). MR 31:2243

Department of Mathematics, Seoul National University, Seoul 151-742, Korea

E-mail address: chkang@math.snu.ac.kr 